Original Research Article

\title{
Comparative analysis of efficacy and adverse effects of tacrolimus vs cyclosporine in chronic dry eye disease: a tertiary care hospital based study
}

\author{
Zeenat Fatima $^{1 *}$, Rakesh Chandra Chaurasia1, Satya Prakash Singh ${ }^{2}$
}

\begin{abstract}
${ }^{1}$ Department of Pharmacology, ${ }^{2}$ Department of Ophthalmology, Motilal Nehru Medical College, Allahabad, Uttar Pradesh, India
\end{abstract}

Received: 10 September 2018 Accepted: 05 October 2018

*Correspondence to:

Dr. Zeenat Fatima,

Email: zeenatf007@gmail.com

Copyright: (c) the author(s), publisher and licensee Medip Academy. This is an openaccess article distributed under the terms of the Creative Commons Attribution NonCommercial License, which permits unrestricted noncommercial use, distribution, and reproduction in any medium, provided the original work is properly cited.

\begin{abstract}
Background: Dry eye disease (DED) is the very common and underdiagnosed ocular condition affecting vision, quality of life, and the outcomes of cataract or refractive surgery. Dry eye disease (DED), either alone or in combination with other conditions, is a frequent cause of ocular irritation that leads the patients to seek ophthalmic care. Due to a wide variety of presenting symptoms, it is often unrecognized and this causes great frustration of the patient and treating physician. While these symptoms often improve with appropriate treatment, usually in majority of the cases the disease may not be curable. Aim of the study was to compare the efficacy of tacrolimus and cyclosporine in dry eye disease.

Methods: This was a single centred, 3 months prospective study. Patients with unilateral or bilateral dry eye disease and an ocular surface disease index score $>12$, atleast one eye with schirmer score $<5 \mathrm{~mm}$ and TBUT $<10$ s were enrolled in the study. The enrolled patients were randomly divided into two groups, twenty-five patients in Group 1 and twenty three patients in Group 2 completed the follow up. Group 1(n=25) who received $0.03 \%$ tacrolimus eye ointment twice daily for consecutive 3 months and Group $2(n=23)$ received $0.05 \%$ cyclosporine eye drops twice daily for consecutive 3 months the primary efficacy outcome was Schirmer score after 3 months. The secondary outcomes were TBUT and adverse effects.
\end{abstract}

Results: After 3 months, both the treatment groups showed significant improvement in mean Schirmer score $(\mathrm{p}<0.001)$ and mean TBUT $(\mathrm{p}<0.0001)$. However, on comparing both the groups, mean Schirmer score and mean TBUT, results were comparable. No patient discontinued treatment because of minor ocular adverse effects.

Conclusions: Dry eye patients demonstrated improvement in Schirmer score and TBUT after 3 months of treatment with tacrolimus $0.03 \%$ ointment and cyclosporine $0.05 \%$ eye drops.

Keywords: Cyclosporine, DED, Schirmer score, Tacrolimus, TBUT

\section{INTRODUCTION}

Dry-eye disease (DED) is a multifactorial disease of the tears and ocular surface which is associated with either increased tear film evaporation on the surface of the eye or decreased tear production by the meibomian glands. It is a pathological condition where the lacrimal functional unit is altered. This unit involves the lacrimal glands, ocular surface, lids and the sensory and motor nerves that connect them. ${ }^{1}$ The major components of the tear film itself are controlled by this functional unit, and respond to several factors like environmental, endocrinological, and cortical influences. The final aim is to maintain tears integrity, transparency of the cornea, and the quality of the image projected onto the retina. ${ }^{1-3}$ The tear film consists of 
multiple layers that lie upon the ocular surface, and diverse tear components that maintain ocular surface health. ${ }^{4}$

Dry eye disease results in symptoms of ocular discomfort (such as a burning sensation, itching, redness, stinging, pain, and foreign body sensation), visual disturbance, and tear film instability, with potential damage to the ocular surface. It is accompanied by increased osmolarity of the tear film and inflammation of the ocular surface.

The pathological mechanisms of DED, depending on the disease subtype, may have different etiologies. ${ }^{5}$ Nevertheless, there are two core mechanisms that converge and are interrelated, hyperosmolarity and chronic inflammation. Other mechanisms in parallel, such as preservative toxicity or acute inflammation, may worsen the disease state.

Inflammation of the ocular surface arises as a result of tear hyperosmolarity and other etiological factors, which start an inflammatory cascade in the epithelial surface cells involving MAP kinases, NFאB signaling pathways and production of inflammatory cytokines (IL-1 $\alpha ;-1 \beta$; TNF$\alpha)$ and MMPs (MMP9). ${ }^{6,7}$ There is increasing evidence supporting ocular surface stress results in infiltration by autoreactive T-cells.

Experimental models have demonstrated the involvement of infiltrating autoreactive T-cells in the immunopathogenesis of murine dry eye disease. ${ }^{8-10}$ Despite the fact that hundreds of millions of people throughout the world suffer from DED, there is no approved global cure for this condition. Moreover, very few treatments for DED have achieved approval by regulatory agencies, and only in certain countries. Based on the recent advances in the understanding of the physiopathology of DED, a wide variety of treatment strategies have been proposed and developed for addressing unmet needs in the treatment of DED, some of them currently under research. The objective of this research article is to describe and compare the efficacy of two calcineurin inhibitors i.e., cyclosporine and tacrolimus in chronic dry eye disease.

\section{METHODS}

The present study was randomized, prospective, comparative, interventional study carried out on patients who were diagnosed as case of dry eye disease, attending outpatient department at Regional Institute of Ophthalmology (M.D. Eye Hospital), Allahabad during the year June 2016- May 2017, after taking permission from ethical committee of M.L.N. Medical College, Allahabad. Patients attending the outpatient department were screened on the basis of inclusion criteria and those fulfilling the criteria were included in the study.

\section{Inclusion criteria}

- $\quad$ Patients included men and women aged $\geq 18$ years.
- Have symptoms of dry eye disease for $\geq 6$ months in any or both the eyes supported by a previous clinical diagnosis.

- Must be able to understand and follow study related advice.

- Patients reporting no improvement in subjective symptoms in response to artificial tear therapy.

- $\quad$ OSDI score $\geq 12$ at screening.

- Following signs at screening and baseline visits in atleast one eye:

- Tear film break up time $\leq 10 \mathrm{~s}$.

- Schirmer tear test without anesthesia $\leq 5 \mathrm{~mm}$ in 5 minutes.

\section{Exclusion criteria}

- $\quad$ Not willing to give consent.

- Active blepharitis, meibomian gland disease, lid margin inflammation or ocular allergy.

- Any structural abnormalities on external eye examination for e.g., entropion, trichiasis, lid scarring and many more.

- Any inflammation or active structural changes in the iris or anterior chamber.

- $\quad$ Single functioning eye.

- Glaucoma.

- $\quad$ Previous eye surgery or punctual occlusion 6 months before study entry.

- Any systemic or topical medication other than artificial tears.

- Any systemic or topical antibacterial or antiinflammatory drug treatment 30 days before study entry.

- Immunosuppressive systemic therapy 90 days before the study entry.

- Contact lens wearer.

- Presence of any corneal infection or any corneal disease (marginal ulcer, opacity, scar, bullouskeratopaty, symblepharon or tumor)

The study consisted of 5 visits conducted during 2 sequential phases i.e., screening/eligibility phase and treatment phase, which included next 4 visits conducted at day 1, 1 month, 2 months, and 3 months. At screening, patients discontinued use of all pre study medications, and the eligibility visit was scheduled after a predetermined washout period according to patient's pre study medication. At the end of the eligibility visit, eligible patients were randomized in a $1: 1$ ratio by assigned number and the criteria as described above in two Groups (1 and 2) and started treatment with:

- $\quad$ Patients assigned in Group 1 were treated with $0.03 \%$ tacrolimus ointment.

- Patients assigned in Group 2 were treated with $0.05 \%$ cyclosporine eye drops.

According to the treatment allocated, patients were advised to instill $0.03 \%$ tacrolimus ointment and $0.05 \%$ 
cyclosporine eye drops twice daily to each eye as monotherapy. The need for at least a 3-month trial period to fully gauge the effectiveness of this therapy was stressed to each patient. All patients were instructed not to use any other topical ophthalmic medications, other than given medication during the study period. Patients were instructed to visit after 1 month, 2 months, and 3 months after starting treatment for the subsequent follow up visits, which was scheduled for each patient. At each study visit, an interval medical history was obtained, and any side effects were assessed, an ophthalmic examination including slit lamp biomicroscopy of the anterior eye segment, Schirmer test, tear film breakup time (TBUT) was performed and the OSDI questionnaire was completed. Patients were instructed to instill the allocated drug in both eyes twice daily for consecutive 3 months, unless a safety issue prevented instillation and to contact us if any problems arise. Patients were also advised that burning on application of cyclosporine and tacrolimus is common and also to avoid sun exposure following instillation of tacrolimus.

In each study visit TBUT was evaluated first, followed by Schirmer tear test.

\section{Tear film breakup time}

Test was conducted at room temperature with fans switched off, and all readings were taken by a single observer. We performed this test by moistening a fluorescein sodium strip with sterile normal saline and applying it to the inferior fornix. After several blinks, patient was instructed not to blink further, and the tear film was examined using a broad beam of the slit lamp with a blue filter. The time lapse between the last blink and the appearance of the first randomly distributed dark discontinuity in the fluorescein stained tear film was taken as the TBUT. TBUT was measured before conducting other tests. TBUT was performed three times and mean of the readings was noted. Patients with TBUT <10 s were included in the study.

\section{Schirmer tear test}

Schirmer I without anaesthesia using a filter paper, Whatman No.41, 5mm in width and $35 \mathrm{~mm}$ in length, to quantify basal and reflex tear productions in a unit of time. The paper is carefully placed on the junction of the middle third and the lateral margin of the lower eyelid towards the temporal angle. In this study we used Schirmer without anaesthesia to measure the basal as well as reflex tear production. The length of the moistened portion of the strip was recorded as Schirmer score to an accuracy of $0.5 \mathrm{~mm}$. Patients showing $\leq 5 \mathrm{~mm}$ wetting of the strip were included in the study.

\section{Efficacy assessment}

Efficacy was evaluated primarily and secondarily with an objective measure. The primary objective outcome was
Schirmer score which was taken at each study visit. Secondary objective outcome was TBUT at each followup visit.

\section{Safety assessment}

The safety outcome was measured as the incidence of adverse reactions and the nature of adverse reactions, determined at various visits by means of physical signs and symptoms, external eye examination, slit-lamp microscopy, visual acuity, intraocular pressure, and funduscopy. All patients were also questioned regarding any ocular symptoms related to the study medications by phone calls as well as at all follow up visits.

\section{Statistical analysis}

Data were expressed as means \pm SD. In all analysis, $p<0.05$ was taken to indicate statistical significance for each time interval of 1 month, 2 months, and 3 months. All data were summarized using frequency distributions and/or descriptive summary statistics (mean and standard deviation [SD]). The efficacy analysis population included all patients who completed the study. The safety analysis population included all patients who were enrolled in the study. All statistical analyses included data for the selected eye. Comparisons were made using the paired Student's $t$ test within treatment groups over different time periods and unpaired Student's t test was used for comparison between treatment groups for continuous variables. Chi square test $\left(\chi^{2}\right)$ was used to analyse the categorical variables. All tests were two tailed, with a significance level of 0.05. All statistical analyses were performed using SPSS software version 20.

\section{RESULTS}

A total of 57 patients participated in the study, 30 patients were randomised and put into Group 1 and 27 patients in Group 2. Of which 5 patients were lost to follow up in Group 1 and 4 patients were lost in Group 2. Mean age in Group 1 was $51.92 \pm 8.86$ yrs and for Group 2 was $52.39 \pm 8.10 \mathrm{yrs}$. The overall frequency of males was higher than females with slightly higher frequency of males in cyclosporine group $(69.56 \%)$ than tacrolimus group (61.5\%).

Efficacy was evaluated primarily with Schirmer score and secondarily with TBUT.

\section{Schirmer Score}

Schirmer's tear test was done in all patients. Baseline Schirmer score for treatment Group 1 was $3.02 \pm 1.19 \mathrm{~mm}$ and in Group 2 was $2.67 \pm 1.16 \mathrm{~mm}$ respectively. On follow up in Group 1 Schirmer score has increased to 5.06 \pm 1.65 $\mathrm{mm}$ after 1 month, $6.64 \pm 2.24 \mathrm{~mm}$ after 2 months and $8.58 \pm 2.98 \mathrm{~mm}$ after 3 months (Figure 1). Increase in Schirmer score was statistically significant in this arm on follow-up ( $\mathrm{p}<0.001)$. Similarly, in Group 2, it was found 
that the Schirmer Score (in $\mathrm{mm}$ ) hasincreased to 5.33 \pm 1.99 after 1 month, $6.98 \pm 2.19$ after 2 months and 9.26 \pm 2.90 after 3 months of treatment. The increase in Schirmer score (mm) was highly significant in this arm also, on follow up $(\mathrm{p}<0.001)$.

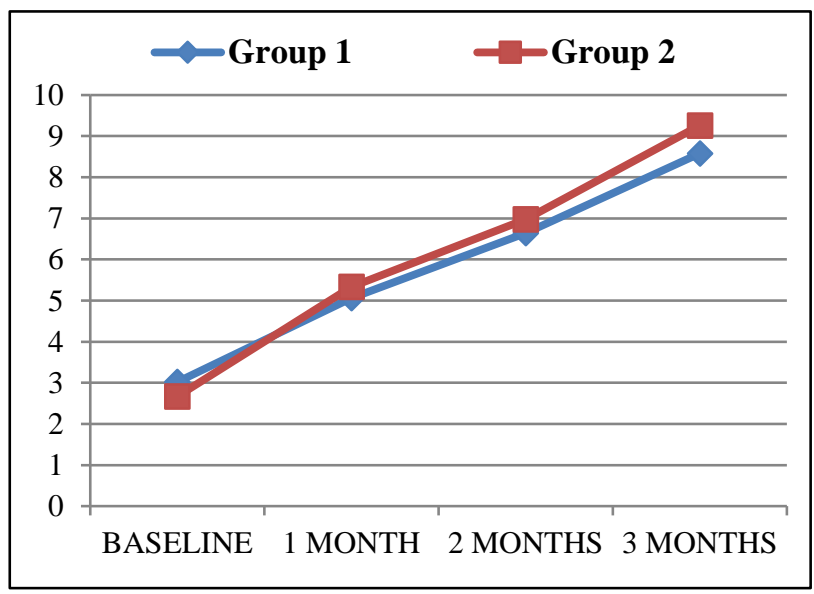

\section{Figure 1: Mean Schirmer Score at different visits in} treatment Group 1 and 2.

Further, on comparing the effects of two drugs resulting in increase in Schirmer score, on follow up, in Group 1 and Group 2, it was found that the increase in Schirmer score was higher in Group 2 than Group 1, however, statistically, the results at follow up visits, were comparable in both groups (Table 1).

Table 1: Comparison of increase in Schirmer Score between Groups at different visits (mean $\pm S D)$.

\begin{tabular}{|llll|}
\hline $\begin{array}{l}\text { Schirmer Score } \\
(\mathbf{m m})\end{array}$ & Group 1 & Group 2 & $\begin{array}{l}\text { p } \\
\text { value }\end{array}$ \\
\hline Baseline & $3.02 \pm 1.19$ & $2.67 \pm 1.16$ & 0.313 \\
\hline 1 month & $5.06 \pm 1.65$ & $5.33 \pm 1.99$ & 0.619 \\
\hline 2 months & $6.64 \pm 2.24$ & $6.98 \pm 2.19$ & 0.600 \\
\hline 3 months & $8.58 \pm 2.98$ & $9.26 \pm 2.90$ & 0.427 \\
\hline
\end{tabular}

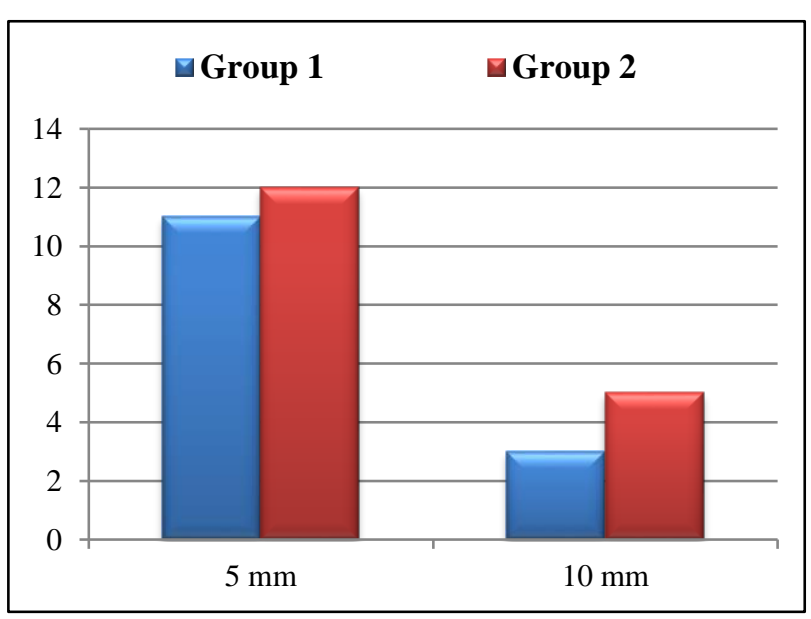

Figure 2: No. of patients showing $5 \mathrm{~mm}$ and $10 \mathrm{~mm}$ improvement in Group 1 and 2.
The two drugs produced similar efficacy outcome statistically. Authors have also observed the responder rate in terms of number of patients who achieved atleast $5 \mathrm{~mm}$ and $10 \mathrm{~mm}$ improvement in Schirmer score after completion of treatment i.e., 3 months and found that it was comparable for each treatment groups. For $5 \mathrm{~mm}$ improvement, it was 44\% (11 patients) for Group 1 and, $52.17 \%$ (12 patients) for Group 2 and for $10 \mathrm{~mm}$ improvement it was $12 \%$ (3 patients) for Group 1 and $21.8 \%$ (5 patients) for Group 2 (Figure 2).

Secondary efficacy outcome was measured in both the groups, in terms of changes in TBUT(secs) and were also compared between the groups.

\section{TBUT}

TBUT(sec) has increased to $7.50 \pm 2.32$ after 1 month, $9.06 \pm 2.64$ after 2 months and $10.50 \pm 3.16$ after 3 months post-treatment from the baseline value of $5.68 \pm 2.03$ in Group 1. The increase in TBUT was statistically significant on follow up in Group 1, ( $p<0.0001)$. Similarly, in Group 2, TBUT values has increased to $7.74 \pm 2.09$ after 1 month, $9.30 \pm 1.99$ after 2 months and $10.61 \pm 1.94$ after 3 months from the baseline value of $5.83 \pm 1.74$. The increase in mean TBUT was statistically significant in Group 2 also, on follow up ( $\mathrm{p}<0.001)$.

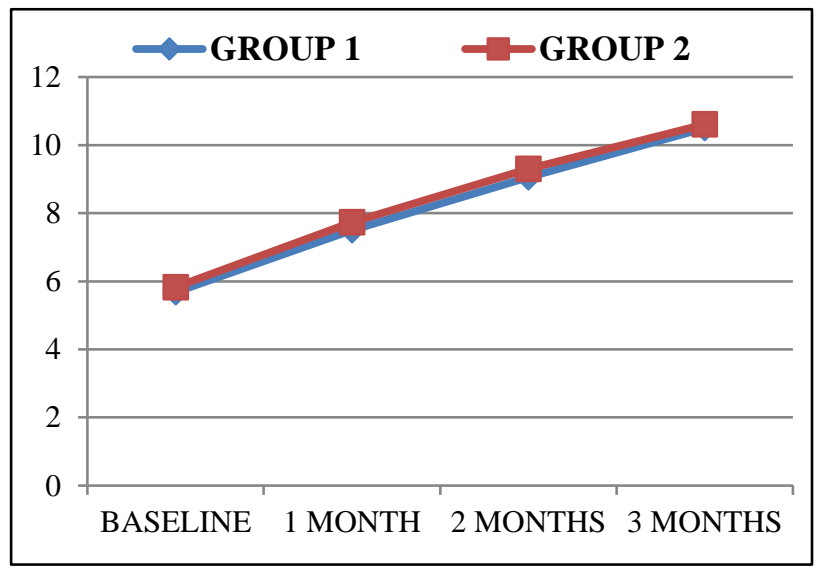

Figure 3: Mean TBUT at different visits in treatment Group 1 and 2.

Table 2: Comparison of TBUT at different visits between Group 1 and 2.

\begin{tabular}{|llll|}
\hline TBUT (secs) & Group 1 & Group 2 & p value \\
\hline Baseline & $5.68 \pm 2.03$ & $5.83 \pm 1.74$ & 0.789 \\
\hline 1 month & $7.50 \pm 2.32$ & $7.74 \pm 2.09$ & 0.709 \\
\hline 2 months & $9.06 \pm 2.64$ & $9.30 \pm 1.99$ & 0.718 \\
\hline 3 months & $10.50 \pm 3.16$ & $10.61 \pm 1.94$ & 0.885 \\
\hline
\end{tabular}

On comparing the two treatment groups, authors found that the baseline values of mean TBUT in both groups were statistically insignificant $(\mathrm{p}=0.0789)$. After 1 month, 2 months and 3 months, TBUT increased in both the 
groups significantly (Figure 3). However, on comparing the increase in TBUT, after 1 month, 2 months and 3 months of follow up between groups, the increase in mean TBUT (secs) was more in Group 2, however the difference was insignificant statistically (Table 2).

\section{Safety}

Incidences of non-serious adverse effects were also reported in this study. No ocular infection was reported during the treatment period. No serious adverse effects warranting the discontinuation of treatment was reported.

Although there were no safety concerns on biomicroscopy or visual acuity testing, and no anatomical changes were observed, non- serious treatment related ocular adverse effects were reported in this study comparable with other previous studies .The most common treatment related ocular adverse effect observed in Group 1 was ocular burning followed by ocular irritation followed by ocular pain .The most frequent adverse effect in Group 2 was also burning sensation on instillation of eye drops. Next common adverse effect reported in Group 2 was conjunctival hyperemia.

\section{DISCUSSION}

Although often disregarded as a minor problem, dry eye is a growing public health concern. Dry eye disease continues to be a challenging disease and its therapy depends on its severity. Recommended treatment for mild dry eye disease is life style changes and use of artificial tears. As it is widely known that inflammation has main role in the etiopathogenesis of dry eye so a number of antiinflammatory treatments are currently in use for its management. Anti-inflammatory medications are considered to be the first line approach in the treatment plethora of dry eye disease.

Topical cyclosporine has been used successfully in the treatment of a number of ocular surface diseases in addition to dry eye. Studies have demonstrated that topical cyclosporine reduces contact lens intolerance in contact lens wearers with dry eye and improves signs and symptoms in patients with superior limbic keratoconjunctivitis as well as ocular rosacea. ${ }^{11-13}$ Topical cyclosporine has also been shown to be an effective a treatment for vernal and atopic keratoconjunctivitis. ${ }^{14,15}$

Several clinical trials have shown the beneficial effects of systemic and topical tacrolimus in the treatment of refractory ocular surface inflammation due to ocular pemphigoid and corneal graft rejection, mooren ulcer, atopic keratoconjunctivitis Moscovici et al, studied the efficacy of tacrolimus for the first time in humans. ${ }^{16-18}$

In a three months trial in 8 dry eye patients, Moscovici et al reported improvement in all patients at the end of treatment in subjective symptoms, ocular surface staining and tearfilm stability. ${ }^{19}$
Authors have found significant increase in Schirmer score for both the treatment groups from 1 month onwards till the end of the study i.e., after 3 months. At the end of the study greater increase in mean Schirmer score was observed in the group treated with cyclosporine than those treated with tacrolimus but difference was statistically insignificant. At the end of the study both tacrolimus and cyclosporine produced comparable improvement in Schirmer score suggesting increased quantity of tear production in both the groups. Result of our present study is comparable with the previous individual studies. $0.03 \%$ tacrolimus and $0.05 \%$ cyclosporine eye drops in dry eye patients, which showed significant improvement in the Schirmer score in follow-up visit as compared to the baseline findings and when compared to the control group. However, authors could not find any human study comparing efficacy of cyclosporine and tacrolimus on Schirmer's score.

In this study authors also found that TBUT of both tacrolimus and cyclosporine treated patients were significantly different in all the visits. There was significant increase in TBUT of both the treatment groups i.e., increasing precorneal tear film stability suggesting improved quality of tear production. The TBUT findings at the end of the study of two groups were comparable.

Sanz Marco investigate the efficacy of $0.03 \%$ topical tacrolimus eyedrops for the treatment of dry eye in graft versus host disease (GVHD) patients resistant/intolerant to $0.05 \%$ topical cyclosporine. Suggested that cyclosporineintolerant patients with dry eye associated with GVHD can be effectively treated with topical tacrolimus. ${ }^{20}$ Moscovici et al in a vehicle-controlled study observed that the average TBUT values increases significantly only after 28 days of treatment from $2.46 \pm 3.37 \mathrm{~s}$ to $4.46 \pm 4.26 \mathrm{~s}$ and even more after 90 days $(5.14 \pm 4.02 \mathrm{~s}$;) as compared to baseline (day 0). ${ }^{21}$ Stonecipher et al, reported significant improvements in TBUT after 6 months treatment with $0.05 \%$ cyclosporine when compared with baseline values, mean increase in TBUT was 0.92 seconds from a baseline value of 1.55 seconds in worse treated eyes and 0.78 seconds from a baseline value of 1.40 seconds in fellow treated eyes. $^{22}$

No ocular infection was reported during the treatment period. No serious adverse effects warranting the discontinuation of treatment was reported. Intraocular pressure was within normal limits. Non- serious treatment related ocular adverse effects were reported in this study comparable with other previous studies. ${ }^{19-22}$ Tacrolimus ointment appears to be safe drug as there are no reports of any serious adverse effects associated with its use from earlier studies and even from this study.

This study demonstrates that in patients who have dry eye symptoms and are refractory to standard artificial tear therapy, $0.03 \%$ tacrolimus ointment and $0.05 \%$ cyclosporine eye drops may alleviate signs and symptoms of the disease and no statistical differences were reported 
between the two groups. However, there were several limitations for this study, first limitation is that the interpretation of this study findings is limited by its partially masked study design. One of the limitations was there were no placebo or control group included in the study since it seemed unethical to leave the patients with dry eye disease untreated for such a prolong period of 3 months. Another limitation was concluding that the active ingredient and not the vehicle was responsible for the improvement, enrollment criteria included only those patients who were unresponsive to artificial tears therapy. Therefore, it is less likely that our results are due to the effects of the vehicle. Compliance is a significant problem, most patients complained of moderate burning sensation. The irritation and intolerance may limit the use of this medication in some patient. Power of the study and also the duration of study to ascertain long term effects and safety. Larger groups and longer follow up are needed for acquiring more information.

\section{CONCLUSION}

This study showed that $0.03 \%$ tacrolimus ointment improves symptoms and signs of dry eye disease in patients unresponsive to artificial tear supplementation. Tacrolimus ointment has been safe and effective in this study. This study also shows that $0.03 \%$ tacrolimus ointment is as safe and effective as $0.05 \%$ cyclosporine eyedrops. Further the cost of treatment with tacrolimus is lower than cyclosporine. Therefore, $0.03 \%$ tacrolimus ointment may be a good therapeutic alternative for patients with local or systemic intolerance to cyclosporine, and it may even be a good early treatment in place of cyclosporine, especially if the therapeutic response of cyclosporine is not as good as expected. However, these findings should be confirmed with a randomized, doublemasked, multicentre, placebo-controlled trial.

Funding: No funding sources

Conflict of interest: None declared

Ethical approval: The study was approved by the Institutional Ethics Committee of Motilal Nehru Medical College, Allahabad, U.P, India

\section{REFERENCES}

1. Stern ME, Beuerman RW, Fox RI, Gao J, Mircheff AK, Pflugfelder SC. The pathology of dry eye: the interaction between the ocular surface and lacrimal glands. Cornea. 1998;17:584-9.

2. Pflugfelder SC, Solomon A, Stern ME. The diagnosis and management of dry eye: a twenty-five-year review. Cornea. 2000;19:644-9.

3. Stern ME, Gao J, Siemasko KF, Beuerman RW, Pflugfelder SC. The role of the lacrimal functional unit in the pathophysiology of dry eye. Exp Eye Res. 2004;78:409-16.

4. Asbell PA, Stapleton FJ, Wickstrom K, Akpek EK, Aragona P, Dana R, et al. The international workshop on meibomian gland dysfunction: report of the clinical trials subcommittee. Invest Ophthalmol Vis Sci. 2011;52:2065-85.

5. Bron AJ, Tomlinson A, Foulks GN, Pepose JS, Baudouin C, Geerling G, et al. Rethinking dry eye disease: a perspective on clinical implications. Ocul Surf. 2014;12:S1-31.

6. Li DQ, Chen Z, Song XJ, Luo L, Pflugfelder SC. Stimulation of matrix metalloproteinases by hyperosmolarity via a JNK pathway in human corneal epithelial cells. Invest Ophthalmol Vis Sci. 2004;45:4302-11.

7. De Paiva CS, Corrales RM, Villarreal AL, Farley WJ, Li DQ, Stern ME, et al. Corticosteroid and doxycycline suppress MMP-9 and inflammatory cytokine expression, MAPK activation in the corneal epithelium in experimental dry eye. Exp Eye Res. 2006;83:526-35.

8. Zheng X, de Paiva CS, Li DQ, Farley WJ, Pflugfelder SC. Desiccating stress promotion of Th17 differentiation by ocular surface tissues through a dendritic cell-mediated pathway. Invest Ophthalmol Vis Sci. 2010;51:3083-91.

9. Goyal S, Chauhan SK, El Annan J, Nallasamy N, Zhang Q, Dana R. Evidence of corneal lymphangiogenesis in dry eye disease: a potential link to adaptive immunity? Arch Ophthalmol. 2010;128:819-24.

10. Stevenson W, Sadrai Z, Hua J, Kodati S, Huang JF, Chauhan SK, Dana R. Effects of topical Janus kinase inhibition on ocular surface inflammation and immunity. Cornea. 2014;33:177-83.

11. Hom MM. Use of Cyclosporine 0.05\%ophthalmic emulsion for contact lens intolerant patients. Eye Contact Lens. 2006;32(2):109-11.

12. Sahin A, Bozkurt B, Irkec M. Topical Cyclosporine. A in the treatment of superior limbickera to conjunctivitis: a long-term follow-up. Cornea. 2008;27(2):193-5.

13. Schechter BA, Katz RS, Friedman LS. Efficacy oftopical Cyclosporine for the treatment of ocularrosacea. Adv Ther. 2009;26(6):651-9.

14. Wan KH, Chen LJ, Rong SS, Pang CP, Young AL. Topical Cyclosporine in the treatment of allergic conjunctivitis: a meta-analysis. Ophthalmology. 2013;120(11):2197-203.

15. Zicari AM, Zicari A, Nebbioso M, Mari E, Celani C, Lollobrigida $\mathrm{V}$, et al. High-mobility group box-1 (HMGB-1) and serum soluble receptor for advanced glycation end products (sRAGE) in children affected by vernal keratoconjunctivitis. Pediatric Allergy and Immunology. 2014 Feb;25(1):57-63.

16. Aoki S, Mizote H, Minamoto A, Suzuki M, Mishima HK, Tanaka H, Systemic FK506 improved tear secretionin dry eye associated with chronic graft versus hostdisease. Br J Ophthalmol. 2005;89:243-4.

17. Xie H, Chen J, Lin Y, Liu Y, Ye C, Zhou S. Effect of topical FK506 used alone or combined with keratoplasty on patients with recurrent Mooren's corneal ulcer. Yan ke xue bao Eye science. 2006 Dec;22(4):207-13. 
18. Nivenius E, Van Der Ploeg I, Jung K, Chryssanthou E, van Hage M, Montan PG. Tacrolimus ointment vs steroid ointment for eyelid dermatitis in patients with atopic keratoconjunctivitis. Eye. 2007 Jul;21(7):968.

19. Moscovici BK, Holzchuh R, Chiacchio BB, Santo RM, Shimazaki J, Hida RY. Clinical treatment of dry eye using $0.03 \%$ tacrolimus eye drops. Cornea. 2012 Aug 1;31(8):945-9.

20. Sanz-Marco E, Udaondo P, García-Delpech S, Vazquez A, Diaz-Llopis M. Treatment of Refractory Dry Eye Associated with Graft Versus Host Disease with $0.03 \%$ Tacrolimus Eyedrops. Journal of Ocular Pharmacology and Therapeutics. 2013;29(8):776-83.

21. Moscovici BK, Holzchuh R, Sakassegawa-Naves FE, Hoshino-Ruiz DR, Albers MB, Santo RM, et al. Treatment of Sjögren's syndrome dry eye using $0.03 \%$ tacrolimus eye drop: Prospective double-blind randomized study. Contact Lens and Anterior Eye. 2015 Oct 1;38(5):373-8.

22. Stonecipher KG, Torkildsen GL, Ousler GW, Morris S, Villanueva L, Hollander DA. The IMPACT study: a prospective evaluation of the effects of Cyclosporine ophthalmic emulsion $0.05 \%$ on ocular surface staining and visual performance in patients with dry eye. Clinical Ophthalmology (Auckland, NZ). 2016;10:887-95.

Cite this article as: Zeenat F, Chaurasia RC, Singh SP. Comparative analysis of efficacy and adverse effects of tacrolimus vs cyclosporine in chronic dry eye disease: a tertiary care hospital based study. Int $\mathbf{J}$ Basic Clin Pharmacol 2018;7:2216-22. 